\title{
La salud pública en el continuo salud-enfermedad: un análisis desde la mirada profesional
}

\section{Public health in the health-disease continuum: an analysis from a professional perspective}

\author{
Valeria Santoro-Lamelas
}

\begin{abstract}
Departamento de Psicología Social. Universidad de Barcelona. Barcelona. España. vsantorol@ ub.edu; vasantoro@gmail.com
\end{abstract}

Recibido 15 diciembre 2014/Enviado para modificación 3 julio 2015/Aceptado 18 enero 2016

\section{RESUMEN}

Objetivo Explorar los componentes que las y los profesionales de salud utilizan para construir y delimitar el concepto de salud pública dentro de las políticas públicas de salud.

Método Análisis cualitativo del contenido. A partir de 20 entrevistas semiestructuradas a profesionales de salud participantes del proyecto demostrativo de la Agència de Salut Pública de Catalunya en la Garrotxa, España (periodo 2009-2012).

Resultados Las y los profesionales de salud utilizan el continuo salud-enfermedad para situar a todas las políticas del Estado en materia de salud: sitúan a la salud pública hacia el extremo de salud y a la Asistencia Sanitaria hacia el extremo de la enfermedad. Asimismo, realizan un ejercicio de contraste de la salud pública con la asistencia sanitaria, para definir y delimitar cada una de las áreas de intervención, a partir del foco de abordaje, el objeto de intervención, la demanda, la temporalidad, la invisibilidad y la consistencia.

Discusión El continuo salud-enfermedad es un elemento relevante para situar a las políticas públicas de salud. Es necesario profundizar sobre el concepto de bienestar para actuar bajo una mirada positiva de salud. Asimismo, el reconocimiento de la multiplicidad de elementos que inciden en la salud pública, hacen que se vuelva permeable a las iniciativas de otras instituciones, a la vez que tornan presente a la salud pública en otros espacios de intervención.

Palabras Clave: Salud pública, servicios de salud, salud, enfermedad (fuente: DeCS, BIREME).

\section{ABSTRACT}

Objective To explore the components that health professionals use to build and define the concept of Public Health as a specific area of knowledge and intervention within public health policies. 
Method A qualitative analysis of content was performed based on 20 semi-structured interviews with health professionals participating in the demonstration project conducted by the Public Health Agency of Garrotxa in Catalonia, Spain (2009-2012). Results Health professionals use the health-disease continuum to provide guidance to all State policies on health: public health is usually related to health and care regarding disease.

Also, professionals contrast public health against health care to define and delineate each intervention area based on the approach, the objectives, the demand, the timing, the invisibility and the consistency.

Discussion The continuum health-disease is a relevant element to guide public health policies. It is necessary to develop the concept of wellbeing to act under a positive view of health. Moreover, recognizing the multiplicity of elements that affect public health makes it permeable to the initiatives of other institutions, while they turn to Public Health in other areas of intervention.

Key Words: Public health, health services, health, disease (source: MeSH, NLM).

$\mathrm{E}$ xisten diferentes definiciones del concepto de salud que pueden ser agrupadas en tres grandes bloques: a. La salud pensada desde la enfermedad; b. Trascendiéndola, como "algo más que la ausencia de enfermedad", y; c. Como normatividad (1-3).

Las definiciones de la salud desde la enfermedad son aquellas que describen a la salud en contraposición a los términos negativos asociados al malestar y el desajuste del cuerpo, la mente o las relaciones. Dentro de estas definiciones, las corrientes biologisistas se centran en el componente orgánico (4), mientras que otras agregan a los componentes biológicos factores psíquicos, sociales y materiales (5). En ambas posturas el malestar y la enfermedad son elementos indispensables para definir la salud, siendo conceptualizada como el equilibrio de la persona (en sentido orgánico o más allá de este) que le permite adaptarse a las exigencias del medio.

Los conceptos ampliados de la salud plantean la necesidad de definir a la salud como algo que trasciende la falta de malestar. Se destacan dos definiciones esenciales: aquellas que definen a la salud agregando el componente de bienestar y aquellas que destacan el componente social de la salud (la salud como valor social).

Las definiciones de salud desde el bienestar agregan a la mirada de la salud como ausencia de sufrimiento y/o enfermedad una cara positiva: el bienestar. La enunciación de la OMS es un ejemplo de este planteamiento: [La salud] "es el estado completo de bienestar físico, mental y social, no consiste solamente en la ausencia de enfermedad"' (6). 
Por su parte, las definiciones de la salud como valor social destacan el vínculo entre la salud y la sociedad, entendiendo a la primera como recurso y como el resultado de las relaciones y estructuras sociales (7).

Finalmente, Canguilhem define a la salud como la normatividad, es decir, la capacidad de sobrevivir, afrontar riesgos y establecer nuevas normas (8). En este marco, la salud es entendida como capacidad creativa que excede los márgenes de la adaptación biofísica o psicosocial a las normas socialmente establecidas.

Precisamente, la concepción de salud desde la normatividad es relevante por plantear un cambio de perspectiva respecto de las visiones tradicionales de la salud que configuran su definición a partir de la correspondencia del funcionamiento de un organismo con unas normas: ser normativo implica que el organismo es capaz de vivir de acuerdo con sus propias normas de vida. Para ello, este organismo tiene un amplio margen de libertad en relación a las condiciones y normas definidas por el entorno (9).

Asimismo, Canguilhem, hace una distinción entre la salud y la enfermedad en relación a su visibilidad; mientras que entiende a la enfermedad como un concepto ruidoso, por hacerse visible y presente en el padecimiento de la persona, comprende a la salud como un fenómeno silencioso puesto que nos permite el desarrollo de nuestra cotidianidad sin preocuparnos en ella.

Actualmente, los discursos oficiales de la Salud Pública, toman las definiciones ampliadas de la salud resaltando la importancia del bienestar y el valor social de la salud como sus elementos constitutivos $(10,11)$.

Las definiciones institucionales de la salud y la Salud Pública, dan marcos de interpretación de la realidad del cuerpo, relaciones y las prácticas de cuidado. Ahora bien, siguiendo una perspectiva crítica de los procesos sociales (12), las personas en su quehacer cotidiano re-producen sentidos y prácticas concretas vinculadas con la salud. En este sentido, las y los profesionales de Salud Pública, en el diseño de intervenciones y su práctica profesional, reconstruyen a la salud como área de intervención. Por ello, resulta pertinente conocer qué argumentaciones, opiniones y presupuestos manejan quienes cotidianamente intervienen en Salud Pública.

Bajo este marco, el objetivo del estudio es explorar los componentes que las y los profesionales de salud utilizan para construir y delimitar el con- 
cepto de Salud Pública como área de saber e intervención específica dentro de las políticas públicas de salud.

\section{MÉTODO}

El estudio desarrollado se enmarca desde la perspectiva crítica de la psicología social, entendiendo que los procesos de construcción de sentidos de la Salud se entretejen en marcos sociales e históricos específicos.

Se desarrolla una metodología cualitativa, a partir de la utilización del método lingüístico, el cual considera al lenguaje como componente esencial para el acceso a la producción de significados y para el análisis de enunciados vinculados a la producción de estos (13).

El trabajo de campo se realizó en uno de los proyectos demostrativos llevados a cabo para el despliegue de la Agència de Salut Pública de Catalunya (ASPCAT): el Proyecto demostrativo del ASPCAT de la Garrotxa, en España.

La selección de informantes clave se basó en la identificación de un equipo motor del proyecto, el autodenominado "Equipo Operativo", compuesto por agentes de salud de niveles centrales, regionales y territoriales. Este Equipo Operativo era el encargado de dirigir el curso del proyecto, analizar propuestas de intervención y reformularlas en aras de garantizar la ejecución del proyecto. Así pues, realizaron entrevistas a todos los miembros activos del equipo operativo y a otros agentes que, aunque no formaran explícitamente parte de este equipo, participaban en él de forma directa y activa en él.

Se realizaron 20 entrevistas semiestructuradas a informantes clave. Se escogió esta técnica por permitir captar la información vivida y capturar discursos particulares que hacen referencia a significados socialmente compartidos. Las personas entrevistadas fueron informadas de los objetivos de las entrevistas y el tratamiento de los datos. Todas ellas dieron el consentimiento de la participación en el proyecto.

Las entrevistas tuvieron una duración de entre $45 \mathrm{~min}$ y $1 \mathrm{~h} .15 \mathrm{~min}$. Con la finalidad de hacer un seguimiento a todo el proceso de despliegue del proyecto de Salud Pública, las entrevistas fueron realizadas en 4 fases durante el periodo 2009-2012: [1] inicio y desarrollo del proyecto, [2] Finalización de la fase conceptual del proyecto, [3] Materialización de los resultados en intervenciones y [4] un año después de la finalización del proyecto. Todas las 
entrevistas fueron grabadas en audio y trascritas de forma literal.

Los códigos de clasificación de las entrevistas son:

- Período de realización: Fase 1(F1), fase 2(F2), fase 3 (F3), fase 4 (F4).

- Posición dentro del proyecto: Comisión directiva (CD), Equipo operativo (EO), Grupo de trabajo (GT), Otros (O).

- Área profesional de la persona entrevistada: Salud Pública General (SP), Promoción de la Salud (Pm), Protección de la Salud (Pt),

- Vigilancia Epidemiológica (V), CatSalut (CS), Educación (E)

- Nivel de descentralización de su trabajo: Nivel Autonómico (NA), Nivel Regional (NR) y Nivel Territorial (NT).

- Formación profesional: Biología (B), Docente (D), Medicina (M), Farmacia (F), Veterinaria (V) y Medio Ambiente (MA).

Se realizó un análisis cualitativo del contenido. Una vez trascrita la totalidad del corpus, se realizaron lecturas repetidas de todas las entrevistas; se identificaron unidades de sentido en el texto (codificación abierta de fragmentos del texto que hablaran sobre una misma temática); se identificaron subtemas dentro de cada unidad temática. A partir de la codificación abierta se establecieron categorías y subcategorías mediante el análisis de los significados de cada afirmación. Finalmente, se establecieron relaciones entre categorías y subcategorías, que permitieron la creación de ejes de sentido más amplios.

El presente artículo presenta las enunciaciones que las y los profesionales de salud utilizan para configurar la salud pública como área de saber e intervención específica dentro de las políticas públicas en salud.

\section{RESULTADOS}

Los resultados presentados se estructuran en los segmentos de sentido que se indican a continuación.

- Configuración del continuo salud-enfermedad como tapiz a partir del cual situar a cada una de las acciones de gobierno en salud (Cuadro 1). 
Cuadro 1. Citas de las entrevistas realizadas, referenciadas en el artículo

\begin{tabular}{|c|c|}
\hline $\begin{array}{l}\text { Número } \\
\text { de cita }\end{array}$ & Extracto de las entrevistas \\
\hline Cita I & $\begin{array}{l}\text { "Yo lo definiría la salud pública como el intento de aumentar el bienestar de la } \\
\text { población, el intento, todas aquellas digamos actuaciones que promueven y } \\
\text { consiguen aumentar el bienestar de la población". (F1/EO/Pt/NT/MA Pág. 21) }\end{array}$ \\
\hline Cita II & $\begin{array}{l}\text { "Salud y bienestar. Es decir, el bienestar como una línea basal que tú la tienes } \\
\text { que mantener o mejorar, incluso, ¿no?" (F4/0/SP/NA/M Pág. 1) }\end{array}$ \\
\hline Cita III & $\begin{array}{l}\text { "Al bienestar (...) Como la salud entendida como la máxima capacidad para } \\
\text { operar y para relacionarse con el entorno. (...) Es decir, yo tengo bienestar en la } \\
\text { medida en que tengo todas mis capacidades como ser humano para sentirme } \\
\text { bien y también para actuar con el entorno, alguna cosa como esta. Y eso } \\
\text { diríamos que es bienestar" (F4/O/SP/NA/M pág. 2) }\end{array}$ \\
\hline Cita IV & $\begin{array}{l}\text { "Ganar bienestar y también seguramente hacer una determinada acción preven- } \\
\text { tiva sobre las enfermedades, los problemas de salud que habrá si tú te descuidas } \\
\text { la alimentación saludable, ¿no? Porque ahora la alimentación saludable está } \\
\text { claramente vinculada (...) a muchas enfermedades, y a algunas enfermedades } \\
\text { crónicas que tienen en su base una... una causa, digamos, alimentaria (F4/O/SP/ } \\
\text { NA/M Pág. 2) }\end{array}$ \\
\hline Cita V & $\begin{array}{l}\text { "Es decir, que lo que haces (...) sería la atención sobre la historia natural de la } \\
\text { enfermedad (F2/O/SP/NA/M. Pág. 5). }\end{array}$ \\
\hline Cita VI & $\begin{array}{l}\text { "La prevención a lo mejor es un territorio compartido con la primaria, con... } \\
\text { Luego está la atención primaria que aborda la gran mayoría de los problemas de } \\
\text { salud y tiene una fuerte capacidad resolutiva, luego tienes la atención especia- } \\
\text { lizada, los socio sanitarios, el acompañamiento a la muerte, en fin" (F2/O/SP/ } \\
\text { NA/M. Pág. 5). }\end{array}$ \\
\hline Cita VII & $\begin{array}{l}\text { "Tenemos que mirar cómo trabajar la primaria para decir qué necesitas de la } \\
\text { salud pública y qué necesitas que te haga la salud pública. Porque a lo mejor las } \\
\text { personas estas m... más individuales ¿a quienes tendremos como referente? (...) } \\
\text { Básicamente tendrán su médico, su centro de salud, y su médico de cabecera, la } \\
\text { enfermera. Y esta será la que le dará un consejo." (F4/O/SP/NA/M. Pág.6) }\end{array}$ \\
\hline Cita VIII & $\begin{array}{l}\text { "Como salud pública interesa el colectivo, no me interesan las personas" (F4/ } \\
\text { OSP/NA/M. Pág. 7). }\end{array}$ \\
\hline Cita IX & $\begin{array}{l}\text { "El peso o lo que nos viene derivado de la epidemia del sobrepeso" (...) Escucha, } \\
\text { no bebas alcohol (...) una sociedad que consume menos alcohol a lo mejor } \\
\text { está... está mejor"(F4/OSP/NA/M. Pág. 6) }\end{array}$ \\
\hline Cita X & $\begin{array}{l}\text { "Quiero decir ¿cuál es ahora el gran problema de las vacunas de que un niño no } \\
\text { se vacune? ¿Es el niño que puede coger la enfermedad? No. Es que dentro de } \\
\text { una población vacunada estás introduciendo un elemento no vacunado (...) en } \\
\text { realidad lo que estamos diciendo es que tenemos que proteger la comunidad". } \\
\text { (F3/O/Pt/NR/V. Pág. 14) }\end{array}$ \\
\hline Cita XI & $\begin{array}{l}\text { "Miraría, para ser más efectivo, más productivo, más... eh... mirar cuáles son las } \\
\text { causas que determinan tus problemas, y abordar estos. (...) esta es la dimensión } \\
\text { de una persona que trabaja en un servicio de Salud Pública. Mirar cuáles son... } \\
\text { sobre qué actuación puedo incidir de una forma más... más ventajosa sobre el } \\
\text { conjunto de los individuos, y por lo tanto sobre la comunidad” (F4/0/SP/NA/M } \\
\text { Pág. 5) }\end{array}$ \\
\hline Cita XII & $\begin{array}{l}\text { "la acción sobre la salud pública es una agencia que desborda las posibilidades } \\
\text { del Departamento de Salud, tenemos aquel cuadro de los determinantes de salud } \\
\text { he nuevamente?, las acciones que tiene que hacer la salud pública (...) va a tener } \\
\text { que contar con la participación de otros sectores (...) porque si no, no vamos a } \\
\text { Ilegar" (F4/O/SP/NA/M Pág. 11) }\end{array}$ \\
\hline
\end{tabular}




\begin{tabular}{cl}
\hline $\begin{array}{c}\text { Número } \\
\text { de cita }\end{array}$ & \multicolumn{1}{c}{ Extracto de las entrevistas } \\
\hline Cita XIII & $\begin{array}{l}\text { "Salud Pública como una parte de las prestaciones del sistema de salud, que se } \\
\text { producen, ya lo hemos dicho otras veces, en ausencia de demanda, con lo cual } \\
\text { los poderes públicos (...) han de, proactivamente proporcionarlas a la población" } \\
\text { (F2/O/SP/NA/M Pág. 1). }\end{array}$ \\
"Pasa que también siempre hemos tenido claro que esto es un tema de largo \\
plazo, quiero decir que esto se empieza y... sí que irá bien para los alumnos que \\
empiecen a hacer actividades o entrar en este plan desde P3, porque cuando \\
salgan...cuando tengan 16 años (F3/EO/SPiE/NT/NS. Pág 2)
\end{tabular}

Los profesionales de salud entrevistados utilizaron al continuo saludenfermedad para pensar y situar a cada una de las intervenciones desarrolladas dentro del Sistema Público de Salud. En su extremo positivo, ubican a las actuaciones de salud orientadas a alcanzar y mantener un estado óptimo de desarrollo: el bienestar y, en su extremo negativo, localizan a las intervenciones orientadas a evitar o tratar o disminuir las consecuencias de la enfermedad. Concretamente, sitúan a la Salud Pública en los extremos positivos del continuo salud-enfermedad, a partir de su identificación en acciones orientadas a favorecer las condiciones de salud, evitar que se produzca la enfermedad y potenciar el bienestar poblacional (I).

Aunque el bienestar es constituido como el estado deseable para toda la población a alcanzar mediante las políticas de Salud Pública, las y los profesionales de salud presentan ambigüedades en el momento de definir el sentido que atribuyen al concepto de bienestar. En los intentos de acotar su definición, el bienestar es configurado en dos sentidos: (a) como estado basal que permite el desarrollo de las actividades cotidianas. Siendo el 
deber de las y los interventores en salud controlar aquellos factores que podrían distorsionarlo y buscar su mejora. Y (b) a partir de la autorrealización. Es decir, hacia la consecución un estado que permita a la persona alcanzar su potencial (II,III).

Ahora bien, aunque a nivel conceptual el Bienestar excede a la ausencia de la enfermedad, en la práctica interventiva, las y los profesionales de salud relacionan constantemente la promoción del bienestar con actividades dirigidas a evitar las situaciones y prácticas que predisponen al desarrollo de enfermedades. Así, al pensar las intervenciones de salud, los argumentos del bienestar como extremo de salud se asocian su extremo opuesto: la enfermedad. En este sentido, ante la patología, las acciones de gobierno desplazan su foco de intervención a evitar la aparición de causas y factores que predispongan la enfermedad, dejando la promoción del bienestar relegado a un segundo plano, como efecto colateral de la prevención de la enfermedad (IV).

La ubicación de la Salud Pública hacia el extremo de salud, llevaba asociada, la atribución de la especialidad en el tratamiento de la patología (el extremo enfermedad) a otro espacio de intervención de salud: la Asistencia Sanitaria. Abastando entre ambos espacios el total del continuo, de las condiciones posibles de existencia de las personas en relación a su saludenfermedad $(\mathrm{V})$.

- Contrastes y efectos de la construcción de la salud pública en relación con la atención sanitaria

Si bien la diferenciación entre la Salud Pública y la Asistencia Sanitaria no fue discutida conceptualmente, las y los profesionales de salud destacaron dos aspectos que hacen que esta distinción sea limitada en la práctica profesional:

- Pensar los fenómenos de salud-enfermedad en clave de continuo: Expresaron que la diferenciación institucional entre la Asistencia Sanitaria y la Salud Pública se vuelve difusa en la práctica profesional, en especial en aquellas intervenciones que se sitúan en el medio del continuo. Destacaron como principal espacio de indefinición a la prevención (VI).

- La importancia de la Asistencia Sanitaria como el espacio institucional de vinculación con la población: Afirmaron que la Asistencia Sanitaria se vuelve una herramienta de relación entre las 
políticas de salud y la población, a partir del cual la Salud Pública puede hacer llegar sus discursos y detecta problemáticas (VII).

En este escenario, las y los profesionales utilizaron una serie de componentes de contraste, que buscaban salvar los problemas indefinición de los límites entre la Salud Pública y la Asistencia Sanitaria en el continuo saludenfermedad, al mismo tiempo que fueron configurando una imagen compleja y especializada del espacio de saber e intervención en Salud Pública. Estos componentes son:

Foco de abordaje

Las y los profesionales de Salud Pública, plantearon que el abordaje en Salud Pública se hace bajo una visión de interés poblacional, en contraposición con la asistencia sanitaria, que vincularon con la atención individual. así pues, expresaron que la salud pública cambia la lente de cómo conocer e interpretar los problemas de salud, de una cuestión personal a una cuestión colectiva (VIII).

Ahora bien, las personas entrevistadas argumentaron que además del tratamiento poblacional de los temas de Salud Pública, la incidencia hacia la persona adquiere relevancia puesto que por una parte, la suma de cada componente de la comunidad dan como resultante una sociedad más o menos sana (IX) y por otra parte, la persona particular puede ser un foco de transmisión del problema de salud para el resto de la comunidad (X).

Objeto de intervención

Las y los profesionales de Salud Pública expresaron que, dentro del continuo salud-enfermedad, la Salud Pública se aleja de la enfermedad y de sus causas directas para centrar su atención a aquellos elementos que puedan afectar la salud de la población y derivar en algún problema de salud probable (XI). En sentido opuesto, identificaron a la asistencia sanitaria, como el tratamiento directo de la problemática en salud (enfermedad o causas directas de esta).

Puesto que las y los profesionales de salud argumentaron que el abordaje de las múltiples causas que intervienen de salud-enfermedad de la población desborda las posibilidades de intervención del Departamento de Salud, propusieron una apertura de los límites de la intervención en salud pública: incluyendo, por una parte, al área de intervención de la salud pública a espacios que se escapan el ámbito tradicionalmente atribuido al 
Departamento de Salud (urbanismo, educación, ocio, etc); y por otra parte, que estos espacios de intervención ajenos a salud, participen en el diseño de intervenciones y la ejecución de acciones de Salud Pública: Plantearon pues una acción intersectorial para trabajar los elementos, condiciones y situaciones que puedan afectar a la salud poblacional (XII).

La demanda

Las personas entrevistadas definieron como demanda a la acción de la persona o colectivos que se movilizan hacia la institución pública en búsqueda de un servicio. Es decir, afirmaron que la demanda es un requerimiento que se inicia desde la persona que detecta una necesidad hacia una institución. En contraposición a esta acción, que definieron como propia de la lógica de la Asistencia Sanitaria, las y los profesionales atribuyeron a la Salud Pública la ausencia de demanda. Según esta idea, el quehacer de la Salud Pública se crea a sí mismo: es decir, argumentan que son las instituciones del Estado quienes deben desarrollar un papel activo en la búsqueda de posibles necesidades y asumir la responsabilidad de la intervención sobre las necesidades poblacionales que ellas mismas detectan, sin que la población afectada de tal intervención la haya solicitado (XIII).

\section{La temporalidad}

Las y los profesionales contrapusieron la rapidez los resultados de salud de la asistencia sanitaria (que al situarlos en extremo de la enfermedad les atribuyeron un desenlace en un tiempo corto o mediano) a la Salud Pública. Argumentaron que a medida que las acciones de Salud Pública se alejan de la enfermedad hacia los elementos que podrían predisponerla y/o hacia la salud, la relación entre la intervención y el resultado de ella se contempla como una posibilidad (no una certeza) cuyos efectos se podrían evidenciar en un periodo mediano-largo de tiempo (XIV).

Las y los profesionales de salud afirmaron que la temporalidad actúa como un elemento motivador (o no) hacia las intervenciones. En este sentido, expresaron que el largo plazo de los resultados que atribuyen a la Salud Pública, se vuelve desmotivador a nivel personal-profesional por el hecho de no poder ver las consecuencias de su actuación dentro de su etapa profesional y a nivel político, puesto que limitaría la movilización de las fuerzas políticas que esperan resultados a corto término, según los periodos legislativos (XV). La (in) visibilidad

Las y los profesionales entrevistadas/os conceptualizaron a la Salud Pública como un área de salud invisible a los ojos de la población general. 
Plantearon que en el momento que se aleja de las causas directas de la enfermedad (de la cadena causal y en especial del tiempo entre la acción) la relación entre la política pública de salud y sus efectos se vuelve difuminada a los ojos de sus beneficiarios y beneficiarias (XVI).

En este sentido, plantearon que los mismos conceptos de "multicausalidad" e "inversión futura", utilizados para definir y fundamentar la existencia de Salud Pública, debilitarían su legitimidad en un entorno donde la urgencia, inmediatez y la linealidad de las intervenciones son prioritarias (XVII).

La consistencia

Las y los profesionales de salud, asumieron que la Salud Pública, al trascender los límites de las ciencias médicas y biológicas, se sitúan en los suburbios de la salud, en los límites con otras áreas de saber sin ser central en ninguna.

Así, expusieron que la definición de la Salud Pública como espacio intersticial, que establece conexiones entre áreas, y las atraviesa constantemente, lleva asociado estar "entre". Expresaron también, que tal visión de la Salud Pública como espacio transversal e interconectado, hace que pierda fuerza en su legitimización en relación a otras áreas del saber de salud donde prima la especialización en sí misma (XVIII).

\section{DISCUSIÓN}

Los resultados presentados visualizan, en primer lugar, la importancia que el continuo salud-enfermedad adquiere para situar a las intervenciones de salud dentro del sistema público de Salud. La lógica de este continuo permite comprender cómo el control y el tratamiento del extremo negativo constituyen elementos indispensables para garantizar la salud puesto que, cuando el malestar, la enfermedad o la incapacidad están presentes, la Salud irremediablemente se ve afectada. Por su parte, el carácter difuso de las conceptualizaciones sobre el bienestar, deriva a que las intervenciones de Salud Pública, sostenidas en argumentaciones relativas a elementos objetivables propias de una lógica positivista $(\mathrm{XV})$ como son la evitación o prevención de la enfermedad, se erijan en una demostración incuestionable de la conveniencia las acciones en Salud Pública. Ante este escenario, sería relevante profundizar sobre definiciones operativas (siempre momentáneas y situadas) del bienestar que permitan definir líneas de actuación en salud, para que efectivamente el abordaje de la Salud Pública amplíe su intervención hacia el Bienestar poblacional (I,VIII) y/o la normatividad (II,X) 
En segundo lugar, se evidencia que las y los profesionales en el ejercicio de comparación de la salud pública con asistencia sanitaria, configuran a la Salud Pública desde la multiplicidad, la ausencia de demanda y efectos evidenciables a medio o largo plazo. Elementos que hacen que quienes trabajan en salud pública contemplen su práctica profesional bajo la invisibilidad de la población general. En este sentido, el ruido de la propia enfermedad - presente y visible en el malestar de la persona- se asocia con la visibilidad institucional de quienes trabajan con el cuerpo enfermo: la Asistencia Sanitaria, mientras que el silencio que evoca la salud -el cuerpo en silencio de los órganos y de las relaciones sociales - (III), se asocia con la invisibilidad institucional de quienes la promueven, previenen o protegen a la población de posibles enfermedades: La salud pública.

En tercer lugar, el carácter difuso del bienestar y multifactorial de las causas de las causas de la salud, hacen que todas las prácticas y condiciones de vida (educación, la vivienda, el empleo, el ocio, etc.) puedan ser integradas como parte del discurso de la salud pública en la medida que la acción sobre estos ámbitos, aunque se escapen del ámbito estrictamente sanitario, pueden ser pensadas como facilitadoras o impedimentos para la salud poblacional. Es bajo este argumento que el discurso de la Salud se infiltra en todas las áreas de vida y relación $(\mathrm{XIV}, \mathrm{XV})$. En otras palabras, nada se escapa de la acción de gobierno en Salud, dado que todo puede ser objeto de intervención a partir de su posible efecto positivo sobre bienestar y la salud de las personas que conforman la sociedad.

Finalmente, el fundamento de las acciones de gobierno en la salud en el continuo salud-enfermedad deriva a construir a la Salud como un derecho básico e incuestionable, porque refiere a algo tan esencial y preciado como la propia vida. Es decir, al asumir que tener salud es, por una parte, estar libre de enfermedades que pongan en peligro la supervivencia y de perturbaciones que limiten nuestras actividades cotidianas y, por otra parte, es un estado que permite a la persona y a la comunidad alcanzar su potencial, la Salud es constituida como un valor superior, una condición indispensable para la vida y el desarrollo personal y social (I). Por ello, según la mirada de las y los profesionales, las tecnologías de gobierno justificadas en la Salud adquieren un carácter ético fundamental y se vuelven "intrínsecamente bondadosas".

Agradecimientos: La autora agradece especialmente a Félix Vázquez Sixto, quien ha sido el director de la tesis doctoral de la que deriva este artículo. Asimismo, agradece la colaboración de la Agència de Salut Pública de Catalunya y de las/os profesionales de salud que participaron el estudio. 


\section{REFERENCIAS}

1. Caponi S. Georges Canguilhem y el estatuto epistemológico del concepto de salud. História, Ciências, Saude. 1997; 4(2): 287-307.

2. Greco M. On the vitality of vitalism. Theory Culture \& Society. 2005; 22(1), 15-27.

3. Canguilhem G. Escritos sobre la medicina. Buenos Aires: Amorrortu, 2004.

4. Contandriopoulos A. P. Elementos para una "topografía" del concepto de Salud. Ruptures, Revista Interdisciplinaria de Salud. 2006; 11(1): 86-99.

5. Seth B, Goldsmith SB. The status of Health Status Indicators. Health Service Reports. 1975; 87(3): 212-220.

6. Organización Mundial de la Salud [editorial] Constitución de la Organización Mundial de la Salud. 1946. Documentos básicos, Supl. de la $45^{\circ}$ edición, Octubre de 2006.

7. Bunton R, Macdonald G. (Eds). Health Promotion: Disciplines and Diversity. Londres: Routledge; 1992.

8. Greco M. On the Art of life: a Vitalist Reading of Medical Humanities. En: Latimer J, SchiIImeier M (Eds.) Un/knowing Bodies. Londres: Wiley-Blackwell; 2009.

9. Canguilhem G. Lo normal y lo patológico. Buenos Aires: Siglo XXI; 1971.

10. Catalunya. Llei $18 / 2009$, del 22 d'octubre, de salut pública. Diari Oficial de la Generalitat de Catalunya, 30 d'octubre de 2009, núm. 5495, pp. 81070-81116

11. Organización Mundial de la Salud [Editorial]. Carta de Ottawa para la Promoción de la Salud (1986). Salud Pública y Educación para la Salud. 2001; 1 (1): 19-22.

12. Burr V. The Person in Social Psychology. Hove: Psychology Press; 2002.

13. Elejabarrieta F. El método lingüístico: Técnicas de obtención de información. Departamento de Psicología de la Salud y Psicología Social. Universidad Autónoma de Barcelona; 1997.

14. Foucault M. Historia de la medicalización. Educación Médica y Salud. 1977; 11 (1):1-25

15. Greco M. Thinking beyond polemics: approaching the health society through Foucault. Österreichische Zeitschrift für Soziologie. 2009; 34(2): 13-27.

16. Foucault M. (1978-1979). Nacimiento de la biopolítica. Madrid: Ediciones AKAL; 2009. 